\title{
Römische militärmedizinische Versorgung und Berichterstattung der Soldaten: Ein Vorschlag zur Kategorisierung der Urkunden*
}

\author{
Andrea Bernini / Anna Maria Kaiser
}

\section{Einleitung}

In den letzten Jahrzehnten hat sich ein besonderes Interesse an der antiken Medizin entwickelt, welches sich in verschiedenen Zugangsweisen zur Thematik manifestiert. So stehen bei den Studien Wilmanns' die historisch-archäologischen Quellen im Mittelpunkt, ebenso bei Krause; Wesch-Klein und Hirt Raj fokussieren auf die sozialen Aspekte; Salazar, Draycott und Israelowich rücken die primär medizinischen Belange in den Mittelpunkt. ${ }^{1}$ Im Zuge der rezenten Übersicht über die dokumentarischen papyrologischen Quellen zur römischen militärmedizinischen Versorgung von Kaiser (im Druck) versteht sich der vorliegende Beitrag als Versuch zur Kategorisierung jener lateinischen Dokumente, die Militärpersonal nennen, welches aufgrund von Krankheiten oder Verletzungen medizinische Behandlung benötigt. ${ }^{2}$ Es handelt sich bei diesen Dokumenten um Listen aus der militärischen Verwaltung, deren Inhalt in ganz unterschiedlichem Ausmaß mit der medizinischen Versorgung der betroffenen Soldaten zu tun hat; primärer Zweck des Großteils der Dokumente ist die Feststellung bzw. Weiterleitung der militärischen Stärke.

\section{Kategorisierung}

Die vorgeschlagene Kategorisierung der lateinischen Dokumente zur militärmedizinischen Versorgung fußt auf drei Aspekten:

\footnotetext{
${ }^{*}$ Die Forschung, die zu diesen Ergebnissen geführt hat, wurde vom European Research Council (ERC) im Rahmen des Forschungs- und Innovationsprogramms Horizon 2020 gefördert: ERCPLATINUM-Projekt (grant agreement $n^{\circ}$ 636983) an der Universität Neapel „,Federico II“. Der Beitrag ist die Überarbeitung des Vortrags von A. Bernini und A.M. Kaiser mit dem Titel „P.Masada 723 und die römische militär-medizinische Versorgung“. Alle Zeitangaben sind n. Chr., sofern nicht anders angegeben.

${ }^{1}$ Wilmanns, Rangordnung, Wilmanns, Sanitätsdienst, Krause, Versorgung; Wesch-Klein, Aspekte; Hirt Raj, Médecins; Salazar, Treatment; Draycott, Approaches; Israelowich, Healers.

${ }^{2}$ In diesem Beitrag werden nur jene Texte berücksichtigt, in welchen sich explizite Hinweise auf Kranke befinden, wenn auch in Teilen in Ergänzung.
} 
1. Zweck des Textes: Hier wird unterschieden zwischen medizinischer und militärischer Berichterstattung. Da alle behandelten Texte aus dem militärischen Umfeld kommen, ist der Inhalt per se natürlich militärisch, doch gibt es Dokumente, die nur medizinischen Inhalt aufweisen, während der Großteil der militärischen Listen, welche Kranke oder Verwundete nennen, den rein militärischen Zweck der Ist-Stärke-Feststellung verfolgen und medizinische Belange lediglich als Begründung des militärischen Ausfalls anführen.

2. Beschreibung der Krankheit: Hier erfolgt die Unterteilung der Dokumente danach, ob Therapie(n) oder Pathologie(n) erwähnt werden.

3. Berichterstattung: Hier richtet sich die Unterscheidung danach, ob die kranken Soldaten namentlich genannt werden (individuell) oder ob lediglich die Gesamtzahl der Kranken verzeichnet wird (kollektiv).

Die drei Aspekte beziehen sich auf den Inhalt der Dokumente und nicht auf paratextuale und paläographische Aspekte. Da es sich um militärische Berichte handelt, die zumeist auf die feldverwendbare Gesamtstärke der Mannschaft abzielen, sind die meisten Texte Listen; nur wenige der Urkunden weisen epistulare Merkmale auf. Aus diesen drei Aspekten lassen sich fünf Kategorien ableiten, die nach absteigendem Fokus auf die medizinischen Belange in den Dokumenten wie folgt aussehen (Tabelle 1):

Tabelle 1: Kategorien und Inhalte

\begin{tabular}{|l|l|l|l|}
\hline Kategorien & Zweck & Beschreibung & Berichterstattung \\
\hline A & medizinisch & Therapie & individuell \\
\hline B1 & militärisch & Pathologie & individuell \\
\hline B2 & militärisch & Pathologie & kollektiv \\
\hline C1 & militärisch & --- & individuell \\
\hline C2 & militärisch & --- & kollektiv \\
\hline
\end{tabular}

Im Folgenden sind jene Abschnitte der Texte (im Original und in der Übersetzung), welche die Kranken bzw. die medizinische Versorgung betreffen, fett gedruckt. Die Ausnahme bildet hier P.Masada 723, da der Text komplett zum Militärsanitätswesen gehört.

\subsection{Kategorie A}

Mit Kategorie A haben wir jenes bislang einzigartige Dokument vor uns, das direkt aus der militärmedizinischen Buchhaltung stammt und sich primär, und im heutigen Erhaltungszustand ausschließlich, der medizinischen Versorgung widmet. Der Papyrus wurde während der Grabungen in Masada (geleitet von Yigael Yadin) am 20. November 1963 im sogenannten „Locus of the Scrolls“ gefunden. ${ }^{3}$

\footnotetext{
${ }^{3}$ Siehe Yadin, Excavation, 79-83; Netzer, Masada III, 416-20, wo die Fundstelle „Casemate of
} 
Es ist anzunehmen, dass das Dokument während einer der Belagerungen Masadas geschrieben wurde - damit zeigt es ausgesprochen eindrucksvoll, welche Ansprüche auch in Extremsituationen an die militärische Buchhaltung gestellt wurden. Möglicherweise stammt das Fragment aus dem römischen Lager B, in dem ein Lazarett identifiziert wurde. $\mathrm{Zu}$ welcher Truppe die im Text erwähnten Soldaten gehörten ist nicht mit Sicherheit zu sagen. Insgesamt waren an der Belagerung Masadas etwa 8000 Mann beteiligt - Legionäre und Hilfstruppen. ${ }^{4}$

P.Masada $723^{5}$

\begin{tabular}{|c|c|}
\hline 1 & ]... [ \\
\hline 2 & ] fasc(iae) $\cdot 1[$ [aneae $]$ \\
\hline 3 & ] o [ ] fasc(iae) $\cdot \operatorname{lan}[\mathrm{eae}]$ \\
\hline 4 & ]ẹlio fasc(iae) · lanẹa [e] \\
\hline 5 & ] $\mathrm{i} \cdot \operatorname{numer}($ us) aegr(orum) \\
\hline 6 & aeg(ri) Nicostra[ti] \\
\hline 7 & ]lio aeg(ro) olẹ · cib(arii) (sextans) \\
\hline 8 & Valẹrio aeg(ro) oleị cib(arii) (uncia) \\
\hline 9 & ] aeg(ro) \\
\hline 10 & ] aeg(ro) olei $\cdot \operatorname{cib}($ arii) $($ sextans $)$ \\
\hline
\end{tabular}

... | für ... wollene Bandage | für -us wollene Bandage | für -elius wollene Bandage $\mid{ }^{5} \ldots$ Zahl der Kranken | Kranke des Nicostratus | für -lius, krank, Speiseöl 2 Unzen | für Valerius, krank, Speiseöl 1 Unze | für ..., krank $\mid{ }^{10}$ für ..., krank, Speiseöl 2 Unzen

Obwohl die ursprüngliche Struktur des Textes nicht mit Sicherheit rekonstruiert werden kann, ist der Zweck des Textes eindeutig medizinisch, wenn auch nur im weiteren Sinn, da er sich nicht direkt mit den Kranken selbst befasst oder mit den behandelnden Ärzten oder Sanitätern, sondern Einblick in die buchhalterischen Abläufe und Erfordernisse der militärischen medizinischen Versorgung gibt. Im Dokument gibt es kein Element, das auf die Anwesenheit anderer, jetzt verlorener, nicht-medizinischer Abschnitte hindeutet. Damit kann folgende Textstruktur, bestehend aus mindestens drei Teilen, rekonstruiert werden: 1 . Kategorie der Patienten (Verwundete vs. Kranke); 2. Personen, welche für eine Patientengruppe ver-

\footnotetext{
the Scrolls" genannt wird.

${ }^{4} \mathrm{Vgl}$. Speidel, Einheit, 286.

${ }^{5}$ P.Masada II 723 (= ChLA XLVI 1366; Masada, 73 oder 74); BL X 64. Die hier verwendete Version des Textes ist aus Bernini, Fasce entnommen (siehe dort für weitere Ausführungen).
} 
antwortlich sind; 3. Patientenverzeichnis mit namentlicher Nennung der Kranken und dem ihnen zugewiesenen medizinischen Bedarf. Nicht ausgeschlossen werden kann aufgrund der fragmentarischen Erhaltung des Dokumentes, dass ursprünglich neben den aegri und volnerati weitere Kategorien vorhanden waren, wie etwa die der lippientes von T.Vindol. II 154 (vgl. unten). P.Masada 723 ist als Dokument aus der militärischen Verwaltung ein bisher einzigartiges Dokument, sodass sich keine Möglichkeit zum Vergleich mit ähnlichen Texten bietet. Dokumente, welche explizit Militärärzte belegen, sind hingegen sehr wohl bekannt, im Inhalt jedoch nicht vergleichbar. ${ }^{6}$ Im Falle von P.Masada 723 ist allerdings nicht bekannt, ob es sich bei der namentlich genannten Person, welche für eine Patientengruppe verantwortlich ist, wirklich um einen medicus handelt. Ebenso könnte Nicostratus etwa ein capsarius gewesen sein. ${ }^{7}$

\subsection{Kategorien B1 und B2}

In den Kategorien B1 und B2 befinden sich jene Dokumente, welche nicht primär medizinischen Inhaltes sind, dabei allerdings immerhin noch verschiedene Krankheiten und Krankheitsbilder verzeichnen. Die Krankheit eines Soldaten (individuell beim Namen genannt oder lediglich als Nummer unter vielen) dient dabei als Grund für ein militärisches Handeln - in den hier ausgeführten Beispielen als Grund für die Entlassung aus dem Militärdienst bzw. für die Nichteinsatzfähigkeit der Soldaten.

Ebenso einzigartig wie das Dokument aus Masada ist der Personalakt des Soldaten Sarapion aus dem 4. $\mathrm{Jh}^{8}{ }^{8}$ Dieser Personalakt ist ein bislang singuläres Dokument der römischen Militärverwaltung, das aus den Abschriften dreier Briefe besteht, welche die Laufbahn des Kavalleristen Sarapion beleuchten und in den Jahren 395, 396 und 401 verfasst und an den Kommandanten des Lagers Psophtis, in der Kleinen Oase in Ägypten, adressiert worden waren. Das älteste Schreiben dokumentiert die Beförderung Sarapions vom einfachen Soldaten (miles munifex) zum cataphractarius, einem gepanzerten Reiter, das zweite seine Ernennung zum decurio und das dritte schließlich seine Entlassung aus dem Militärdienst, die missio. In diesem dritten Schreiben werden alle jene genannt, die zusammen mit Sarapion aus dem Militärdienst entlassen wurden; nach Rängen

${ }^{6}$ T.Vindon. 37 (Vindonissa, 30-101); T.Vindon. 69 (Vindonissa, 30-101); CIL XIII 5208,1-2 (= Wilmanns, Sanitätsdienst, 190-1, Nr. 43; Gebenstorf, 1. Jh.), vgl. Wilmanns, Rangordnung, 97100; Speidel, Schreibtafeln, 73 Anm. 16; Wesch-Klein, Aspekte, 79-82. Eine weitere Schreibtafel mit einem Arzt als Empfänger stammt aus dem Hilfstruppenlager Valkenburg in Holland (AE 1975, 634, 2; Davies, Medici, 11, Nr. 5).

${ }^{7}$ Für eine Auflistung der immunes, zu denen auch die medizinischen Chargen gehörten, siehe Dig. 50,6,7 (Tarruttienus Paternus); zur Rangordnung allgemein vgl. Domaszewksi/Dobson, Rangordnung.

${ }^{8}$ ChLA XLIII 1248 (= CPR V 13 = P.Rain.Cent. 165; unbekannter Ort, 401). Vgl. Palme, Legionäre, 78, Nr. 9. 
geordnet, mit der Angabe des Entlassungsgrundes und des Dienstalters. Für die missio causaria, die prinzipiell als ehrenvoll galt, waren eine sorgfältige Prüfung und ärztliche Gutachten notwendig. Derartige Untersuchungen sind hier wohl vorauszusetzen. ${ }^{9}$

\section{Kategorie B1:}

C.Epist.Lat. I $233^{10}$

\begin{tabular}{|c|c|}
\hline 1 & (2. Hand) $\mathfrak{f}(\mathrm{)}) !(\mathrm{r})$ \\
\hline 2 & (1. Hand) Flauius Elẹ[ut]herius $\quad$ H. st . . . . . fṛ̂n(atri) \\
\hline 3 & $\mathrm{p}[\mathrm{rae}] \mathrm{f}(\mathrm{ecto}) \mathrm{k}(\text { astri })^{11} \mathrm{Ps}[\mathrm{oft}(\mathrm{h}) \mathrm{is}]$ \\
\hline 4 & {$[---]$} \\
\hline 5 & {$[---]$} \\
\hline 6 & in ... [ . . . . . . ] tia $\left[-{ }^{-}--\right]$ \\
\hline 7 & nomin[a] $\mathrm{m}[\mathrm{at}$ ]riculis [inserta - - - ex die - - - ] \\
\hline 8 & Vincentio et Fraovitho viris clarissimis consulibus omni armorum genere \\
\hline 9 & quo instructi esse videbantur redhibito eximere curabis \\
\hline 10 & et sunt \\
\hline 11 & dec(uriones) Sarapion colic(us) sti(pendiorum) XVIII \\
\hline 12 & Stefanus debilis sti(pendiorum) XXII \\
\hline 13 & Fileas senex sti(pendiorum) XXXV \\
\hline 14 & Isidorus aegrot(us) sti(pendiorum) XVIII \\
\hline 15 & catafrac(tarii) Doroteus senex sti(pendiorum) XXVII \\
\hline 16 & Foebammon ut s(upra) sti(pendiorum) XX \\
\hline 17 & Apammon colicus sti(pendiorum) $\mathrm{X}$ \\
\hline 18 & Foebammon debil(is) sti(pendiorum) VII \\
\hline 19 & eq(uites) Heraclas senox ${ }^{12}$ sti(pendiorum) XXVII \\
\hline 20 & Ahesis ut s(upra) sti(pendiorum) XXVII \\
\hline 21 & Antonius ut s(upra) sti(pendiorum) XXVII \\
\hline
\end{tabular}

... | Flavius Eleutherius an ... Bruder, | praefectus des castrum Psophtis ... |

$\left.\ldots\right|^{5} \ldots|\ldots|$ in den Matrikeln eingetragenen Namen sollst Du ... unter dem Konsulat der viri clarissimi Vincentius und Fraovithus nach Rückgabe jegli-

\footnotetext{
${ }^{9}$ Graßl, Missio, 281-9; Wesch-Klein, Aspekte, 88-90. Für den Rekrutenbedarf bzw. die Überlebensraten der Soldaten auch in Friedenszeiten vgl. die komplexe Untersuchung von Scheidel, Rekruten, 232-54.

${ }^{10}$ Herkunft unbekannt, 401.

${ }^{11}$ Lies $c$ (astri).

${ }^{12}$ Lies senex.
} 
cher Waffen, mit denen sie ausgestatten waren, streichen lassen. $\mid{ }^{10}$ Die sind: | decuriones: Sarapion, Kolik, 18 Dienstjahre | Stephanus, gebrechlich, 22 Dienstjahre | Phileas, alt, 35 Dienstjahre | Isidorus, krank, 18 Dienstjahre | 15 cataphractarii: Dorotheus, alt, 27 Dienstjahre | Phoebammon, deto, 27 Dienstjahre | Apammon, Kolik, 10 Dienstjahre | Phoebammon, gebrechlich, 7 Dienstjahre $\mid$ equites: Heracles, alt, 27 Dienstjahre $\mid{ }^{20}$ Aphesis, deto, 27 Dienstjahre | Antonius, deto, 27 Dienstjahre

Der Text ist Teil eines liber epistularum; zusammen mit C.Epist.Lat. I 231 und 232 wird Sarapions Karriere nachgezeichnet. Der vorliegende Text beginnt mit einer brieflichen inscriptio und verzeichnet im Anschluss die Namen jener Soldaten, die aus dem Dienst entlassen werden. Die Soldaten sind hierarchisch nach ihren Dienstgraden geordnet; die Entlassungsgründe (Krankheit, Gebrechlichkeit, Alter) werden ebenso angegeben wie die Dienstjahre. Isidorus (Z. 14) wird als aegrotus bezeichnet, weiterer Aufschluss über sein Leiden wird nicht gegeben. Im Gegensatz dazu wird bei Sarapion und Apammon spezifiziert, dass sie unter einer Kolik leiden (colicus, Z. 11; 17). Da der Zweck der Liste die Namhaftmachung der zu entlassenden Soldaten sowie der Entlassungsgründe, zu denen auch Krankheit gehört, ist, und nicht per se die Auflistung von Krankheiten, scheinen die beiden weiteren zur Entlassung genannten Gründe, nämlich debilis und senex (Z. 12; 18 sowie Z. 13; 15; 16; 19-21) nicht als Krankheiten per se zu verstehen zu sein, sondern primär als Gründe der Entlassung, wobei allerdings bei der Gebrechlichkeit auch an die Spezifizierung einer Krankheit gedacht werden könnte, analog zu colicus.

Weder aus dem militärischen Umfeld noch unter den übrigen Dokumenten, welche Kranke nennen, ist ein Vergleichstext zu C.Epist.Lat. I 233 bekannt, doch können ihm zumindest im Hinblick auf die genaue Bezeichnung der einzelnen Krankheiten und Leiden einige Texte aus dem zivilen Umfeld gegenübergestellt werden, die jedoch sämtlich auf Griechisch verfasst sind. Aus der Ptolemäerzeit stammt BGU X 1942, ${ }^{13}$ eine Liste mit Namen von Textilarbeiterinnen, in der mit dem Zusatz ó $\rho \omega \sigma \tau o \varsigma$ Krankheiten vermerkt sind. Mehrere Texte datieren römerzeitlich: O.Claud. II $212^{14}$ ist eine Liste von kranken Arbeitern, die zur familia gehören. Das Dokument gibt an, wer von einem Augenlei-

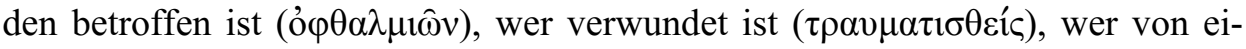
nem Skorpion gebissen wurde ( $\sigma \kappa о \rho \pi เ o ́ \pi \lambda \eta \kappa \tau о \varsigma)$, wer Fieber hat ( $\pi \nu \rho \varepsilon \kappa \tau ו \kappa o ́ \varsigma)$,

\footnotetext{
${ }^{13}$ Unbekannter Ort, Ende 2. - Anfang 1. Jh. v. Chr.

${ }^{14}$ Mons Claudianus, ca. 137-145. Die im Umfeld der Steinbrüche am Mons Claudianus in der Östlichen Wüste in Ägypten gefundenen Listen mit Kranken, bei denen es sich in manchen Fällen ebenfalls um Soldaten handeln dürfte (O.Claud. I, 75-109, bes. 80-81; O.Claud. II, 19-41), listen zumeist lediglich Namen auf, zeigen aber auch, dass einige der Kranken über den Zeitraum von mehreren Monaten (2-5) mit ihrem Gesundheitszustand zu kämpfen hatten.
} 


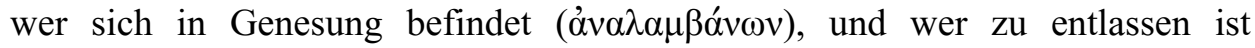

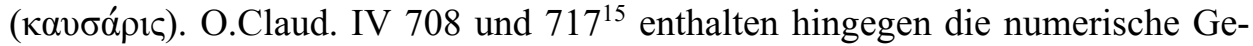
samtzahl der Kranken ( $\alpha \rho\langle\rho\rangle \omega \sigma \tau o l)$ mit genaueren Angaben zur Krankheit, wie dies auch von einer Stärkemeldung der cohors I Tungrorum in Britannien bekannt ist (vgl. Kategorie B2).

\section{Kategorie B2:}

T.Vindol. II $154^{16}$

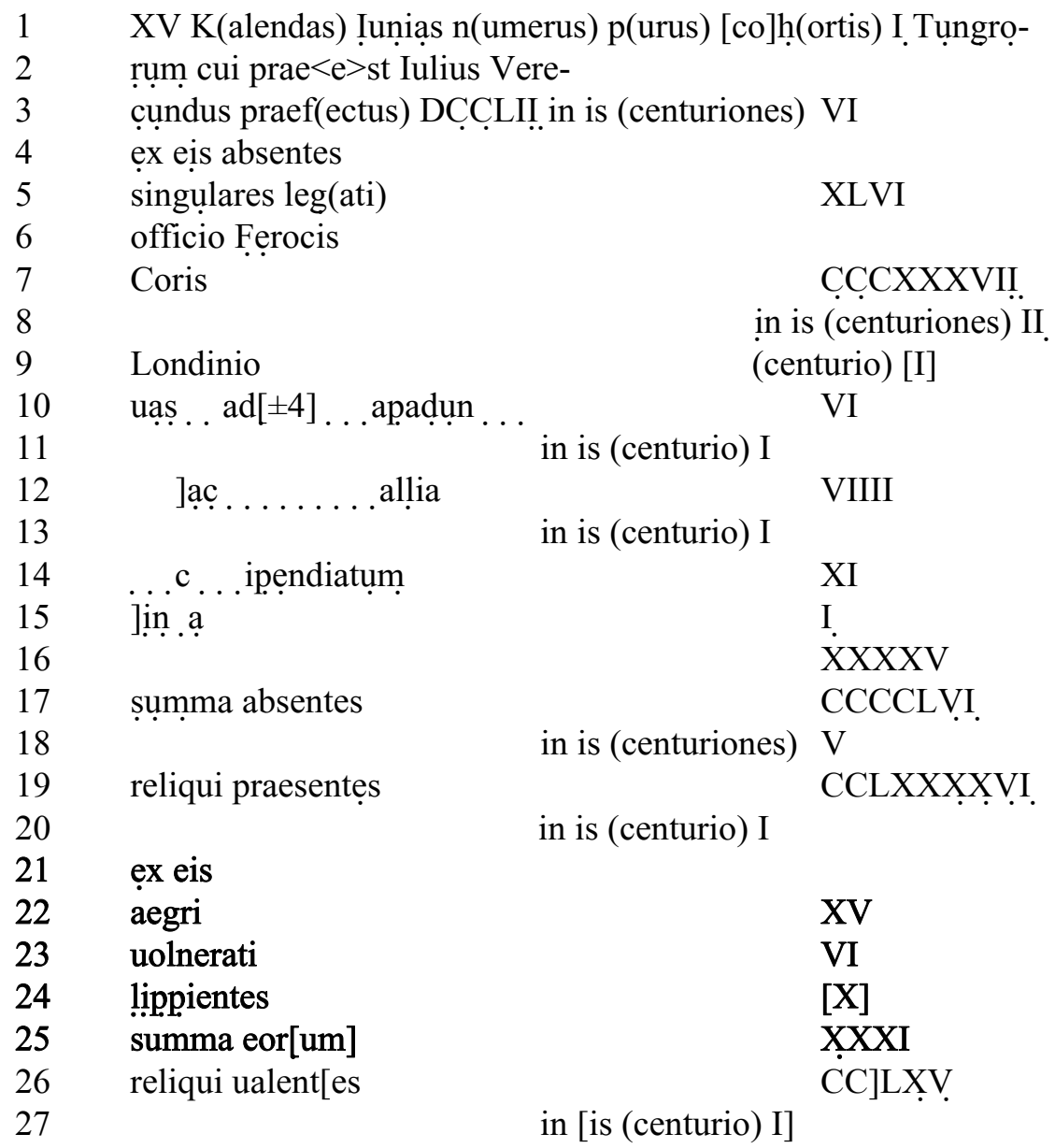

\footnotetext{
15 Mons Claudianus, 98-117.

16 Vindolanda, 92-97.
} 
15. vor den Kalenden des Juni, numerus purus der cohors I Tungrorum, | der Iulius Verecundus, | praefectus, vorsteht: 752 darunter 6 centuriones | von diesen sind abwesend: $\mid{ }^{5}$ Garde des Statthalters $46 \mid$ im officium des Ferox $\mid$ in Coria $337 \mid$ darunter 2 (?) centuriones $\mid$ in London 1 (?) centurio $\left|{ }^{10} \ldots 6\right|$ darunter 1 centurio $|\ldots 9|$ darunter 1 centurio $|\ldots 11|{ }^{15} \ldots 1$ (?) $|\ldots 45|$ Summe der Abwesenden 456 | darunter 5 centuriones | übrig bleiben Anwesende 296 $\left.\right|^{20}$ darunter 1 centurio | von diesen | Kranke 15 | Verwundete 6 | an einer Augenentzündung Leidende $\left.10\right|^{25}$ in Summe 31 | übrig bleiben Dienstfähige 265 | darunter 1 centurio

Der Text lässt sich folgendermaßen strukturieren: 1. Titel der Liste bestehend aus Datum, dem Namen des kommandieren Offiziers und der Gesamtstärke der cohors; 2. Anzahl der Abwesenden mit Beschreibung des Grundes der Abwesenheit bzw. ihrer Dienste; 3. Anzahl der Anwesenden mit Nennung der Kranken sowie der für den Dienst zur Verfügung stehenden gesunden Soldaten. Ana$\log$ zu C.Epist.Lat. I 233 wird bei den Kranken unterschieden zwischen jenen, die schlicht und einfach aegri sind, den Verletzten und den lippientes, die vermutlich an einer Augenentzündung leiden. Bei dem Dokument handelt es sich um eine typische Stärkemeldung einer Truppe, in diesem Fall der cohors I Tungrorum. Die Erwähnung der Kranken dient lediglich dazu festzuhalten, warum jene 31 Mann (4,12\% der Gesamtstärke) nicht für den Dienst zur Verfügung stehen; ${ }^{17}$ medizinischer Zweck lässt sich aus dem Dokument primär keiner ablesen, außer man möchte andenken, dass durch die gesonderte Erwähnung der lippientes besondere Aufmerksamkeit auf die Augenerkrankung gelenkt werden sollte. Als Vergleichsmaterial für diese Stärkemeldung aus Vindolanda können die Ostraka aus Bu Njem herangezogen werden, auch wenn diese Ostraka keinerlei Angaben zum Krankheitsbild der Soldaten verzeichnen und damit in die Kategorien C1 und C2 fallen.

\subsection{Kategorien $\mathrm{C} 1$ und $\mathrm{C} 2$}

In den Kategorien C1 und C2 finden sich schließlich jene Dokumente, welche nicht primär medizinischen Inhalts sind und auch keine Angabe zum Krankheitsbild selbst aufweisen. In der militärischen Administration wurde, wie sich bereits in T.Vindol. II 154 zeigt, selbstverständlich auf die zahlenmäßig exakte Erfassung der verfügbaren, dienstfähigen Soldaten Wert gelegt. Das beginnt bei den sogenannten Morgenmeldungen, die auf der untersten Ebene die Kranken namentlich nennen; so etwa im römischen Lager in Gholaia, in der Africa Pro-

\footnotetext{
${ }^{17}$ Kaiser, Evidenz.
} 
consularis, am Limes Tripolitanus, dem heutigen Bu Njem. Die Krankenrate liegt in diesen Dokumenten zwischen 2,3 und 11,8\%. ${ }^{18}$ Diese Meldungen wurden jeweils eine Ebene höher zusammengefasst und ebenso weitergeleitet, bis am Ende in Bezug auf die Kranken nur mehr die Zahl von Interesse war, nicht mehr ihre Namen und nicht mehr ihre Leiden.

\section{Kategorie C1:}

O.BuNjem 12

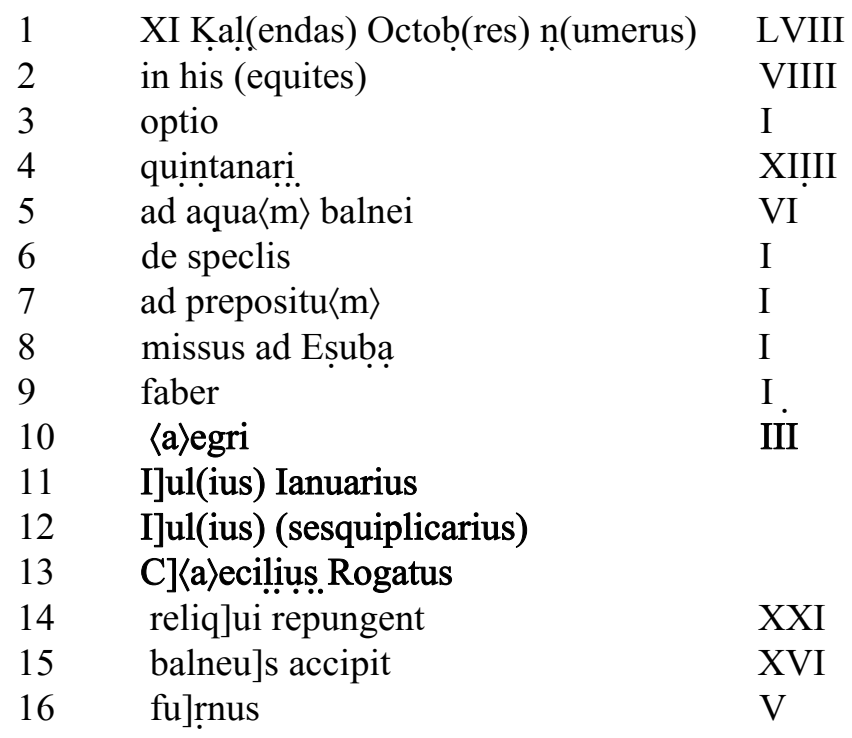

11. vor den Kalenden des Oktober, Zahl 58 | davon Reiter $9 \mid$ optio $1 \mid$ quintanarii $14 \mid{ }^{5}$ für das Badewasser $6 \mid$ Aussichtsposten $1 \mid$ beim praepositus $1 \mid$ nach Esuba geschickt $1 \mid$ Handwerker $1 \mid{ }^{10}$ Kranke 3 | Iulius Ianuarius | Iulius, sesquiplicarius | Cecilius Rogatus | übrig bleiben 21 | ${ }^{15}$ das Bad erhaltend $16 \mid$ Backofen 5

Analog zum oben besprochenen Dokument aus Vindolanda beginnt auch dieser Text mit dem Datum und der Gesamtstärke der Soldaten, welche der nicht näher bezeichneten (Teil)Einheit angehören. Die folgenden Zeilen enthalten die Dienste, die an diesem Tag verrichtet werden, sowie die Zahl der jeweils dazu eingeteilten Soldaten. Im Anschluss daran werden die Kranken genannt - in Summe

\footnotetext{
18 Wesch-Klein, Aspekte, 72 Anm. 3. Für Tagesrapporte, Morgenmeldungen und pridiana verschiedener Größenordnungen und Truppen siehe Speidel, Einheit, 287-300.
} 
drei, und im Gegensatz zu den zu anderen Aufträgen herangezogenen Soldaten werden die Kranken hier auch namentlich genannt. Der Zweck dieser Auflistung ist ebenfalls das Festhalten bzw. die Weitergabe der Gesamtstärke, sowie der Diensteinteilung der Soldaten der (Teil)Einheit. Aus Bu Njem sind weitere 19 Ostraka bekannt, die analog zum hier vorgestellten Beispiel aufgebaut sind und die Namen der Kranken ebenfalls verzeichnen (siehe unten Tabelle 2). ${ }^{19}$

Krankheiten wurden ebenso wie verrichtete Alltagsdienste in täglich geführte Listen eingetragen, wie ein ägyptischer Papyrus aus der Zeit um 81-90 zeigt. ${ }^{20}$ Der Papyrus verzeichnet nach einer Liste von immunes 36 Soldaten namentlich, am linken Rand vertikal, während horizontal die Tagesdaten geführt werden vom 1. bis zum 10. Oktober; dann bricht der Text ab. ${ }^{21}$ In diesem Dokument, das im Layout eine Tabelle ergibt, wurden die Sonderdienste, welche die einzelnen Soldaten an verschiedenen Tagen zu verrichten hatten, eingetragen. So etwa Reinigungs- und Instandhaltungsdienste oder Wachdienste; Soldaten waren zur Reinigung der Latrine und der Unterkünfte abgestellt und versahen Dienst im Lagerbad. ${ }^{22}$ Ein Soldat wurde eingeteilt, um sich um Tragbahren für Verletzte und Kranke zu kümmern; ob zur Herstellung oder Instandhaltung, ist nicht zu sagen. Ein anderer Soldat wird möglicherweise auch in dieser Liste als krank geführt; woran er litt ist auch hier nicht festzustellen. ${ }^{23}$

Parallel zu den oben besprochenen Stärkemeldungen aus Bu Njem sind jene Dokumente zu nennen, deren einziger Unterschied darin liegt, dass die Kranken summarisch, das heißt nicht mehr namentlich, genannt werden. Auch hier stammt der Großteil der erhaltenen Dokumente aus dem Lager in Bu Njem.

\section{Kategorie C2:}

\section{O.BuNjem 5}

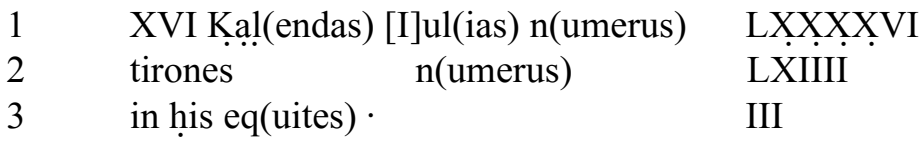

${ }^{19}$ Als Vergleichsbeispiel der familia am Mons Claudianus mag O.Claud. IV 754 (Mons Claudianus, ca. 98-117) dienen, eine Liste von Arbeitern, in welcher zwei Personennamen $\alpha \sigma \theta \varepsilon v \hat{\omega} v$ vorangestellt ist.

${ }^{20}$ Rom.Mil.Rec. 9 (= P.Gen.Lat. 1 = C.Pap.Lat. 106 = ChLA I 7 a, b; unbekannter Ort, um 8190). Vgl. Kaiser, Routinedienste, 115-29.

${ }^{21}$ Liste von immunes: Rom.Mil.Rec. 58 (= P.Gen.Lat. 1 = C.Pap.Lat. 106 = ChLA I 7 a, b; unbekannter Ort, um 81-90).

22 Premerstein, Buchführung, 38; Stauner, Schriftwesen, 20 Anm. 33, mit Verweis auf den Baddienst der Soldaten in Bu Njem.

${ }^{23}$ Rom.Mil.Rec. 9 (= P.Gen.Lat. 1 = C.Pap.Lat. 106 = ChLA I 7 a, b; unbekannter Ort, um 8190). Tragbahre (fercla): M. Antonius Crispus; krank (?) (aeg()): A. Marius Clemens. 


\begin{tabular}{|c|c|c|}
\hline 4 & quintanari & XXII \\
\hline 5 & ad balneu $<$ m $>$ & VIII \\
\hline 6 & de speclis & I \\
\hline 7 & ad praepositu $<$ m $>$ & $\dot{\mathrm{I}}$ \\
\hline 8 & aḍ st(ationem) çamellar(iorum) & I \\
\hline 9 & optio & I \\
\hline 10 & item Rufus & I \\
\hline 11 & $\langle a\rangle$ egri & IIII \\
\hline 12 & excusantur & XXVIII \\
\hline 13 & reliqui munifices & XXVI \\
\hline 14 & b[a]l[n]ẹ[us ac]c̣[ipit & \\
\hline 15 & [furnus & \\
\hline
\end{tabular}

16. vor den Kalenden des Juli, Zahl 86 | tirones Zahl $64 \mid$ darunter Reiter 3 | quintanarii $22 \mid{ }^{5}$ für das Bad $8 \mid$ Aussichtsposten 1 (?) | beim praepositus $1 \mid$ bei der statio camellariorum $1 \mid$ optio $\left.1\right|^{10}$ in gleicher Weise Rufus $1 \mid$ Kranke 4 | Befreite $28 \mid$ übrig im Dienst 26 | das Bad erhaltend ... $\mid{ }^{15}$ Backofen ...

Neben vier lateinischen Ostraka aus Bu Njem fallen weitere zwei Papyri aus dem Beginn des 2. respektive des 3. Jh. in diese Kategorie (Tabelle 2.). ${ }^{24}$

Tabelle 2: Lateinische Urkunden nach Kategorien

\begin{tabular}{|l|l|l|l|l|}
\hline Kategorie & Urkunde & $\begin{array}{l}\text { Herkunft / } \\
\text { Fundort }^{26}\end{array}$ & Datum & Material \\
\hline A & P.Masada 723 & Masada & 73 oder 74 & Papyrus \\
\hline B1 & C.Epist.Lat. I 233 & Unbekannt & 401 & Papyrus \\
\hline B2 & T.Vindol. II 154 & Vindolanda & $92-97$ & Holztafel \\
\hline C1 & O.BuNjem 2 & Bu Njem & $253-259$ & Ostrakon \\
\cline { 2 - 5 } & O.BuNjem 3 & Bu Njem & $253-259$ & Ostrakon \\
\cline { 2 - 5 } & O.BuNjem 7 & Bu Njem & $253-259$ & Ostrakon \\
\cline { 2 - 5 } & O.BuNjem 8 & Bu Njem & $253-259$ & Ostrakon \\
\cline { 2 - 5 } & O.BuNjem 10 & Bu Njem & $253-259$ & Ostrakon \\
\cline { 2 - 5 } & O.BuNjem 12 & Bu Njem & $253-259$ & Ostrakon \\
\cline { 2 - 5 } & O.BuNjem 22 & Bu Njem & $253-259$ & Ostrakon \\
\hline
\end{tabular}

${ }^{24}$ ChLA III 219 (105), C.Epist.Lat. I 191 (3. Jh.). Wiederum gibt es vom Mons Claudianus ähnliche Listen: O.Claud. IV 697, 698, 699 (ca. 98-117) und 721 (138 oder 147-148) nennen unter ầypor die entsprechenden Personennamen; in O.Claud. IV 709, 710 (ca. 98-117) werden die Namen unter der Überschrift $\alpha \dot{\sigma} \theta \varepsilon v 0 \hat{v} \tau \varepsilon \varsigma$ geführt.

${ }^{25}$ O.BuNjem 9, 14 und 29 sind aufgrund ihres Erhaltungszustandes nicht sicher einer der beiden Kategorien $\mathrm{C} 1$ oder $\mathrm{C} 2$ zuzuordnen.

${ }^{26}$ Die Herkunft der Dokumente dürfte vermutliche mit ihrem Fundort gleichzusetzen sein. C.Epist.Lat. I 233, dessen Herkunft unbekannt ist, könnte im Arsinoites gefunden worden sein. 


\begin{tabular}{|l|l|l|l|l|}
\hline \multirow{4}{*}{} & O.BuNjem 25 & Bu Njem & $253-259$ & Ostrakon \\
\cline { 2 - 5 } & O.BuNjem 26 & Bu Njem & $253-259$ & Ostrakon \\
\cline { 2 - 5 } & O.BuNjem 28 & Bu Njem & $253-259$ & Ostrakon \\
\cline { 2 - 5 } & O.BuNjem 30 & Bu Njem & $253-259$ & Ostrakon \\
\cline { 2 - 5 } & O.BuNjem 31 & Bu Njem & $253-259$ & Ostrakon \\
\cline { 2 - 5 } & O.BuNjem 32 & Bu Njem & $253-259$ & Ostrakon \\
\cline { 2 - 5 } & O.BuNjem 33 & Bu Njem & $253-259$ & Ostrakon \\
\cline { 2 - 5 } & O.BuNjem 34 & Bu Njem & $253-259$ & Ostrakon \\
\cline { 2 - 5 } & O.BuNjem 36 & Bu Njem & $253-259$ & Ostrakon \\
\cline { 2 - 5 } & O.BuNjem 42 & Bu Njem & $253-259$ & Ostrakon \\
\cline { 2 - 5 } & O.BuNjem 43 & Bu Njem & $253-259$ & Ostrakon \\
\cline { 2 - 5 } & O.BuNjem 51 & Bu Njem & $253-259$ & Ostrakon \\
\cline { 2 - 5 } & O.BuNjem 55 & Bu Njem & $253-259$ & Ostrakon \\
\hline C2 & ChLA III 219 & Unbekannt & $16 / 09 / 105$ & Papyrus \\
\cline { 2 - 5 } & O.BuNjem 5 & Bu Njem & $253-259$ & Ostrakon \\
\cline { 2 - 5 } & O.BuNjem 6 & Bu Njem & $253-259$ & Ostrakon \\
\cline { 2 - 5 } & O.BuNjem 13 & Bu Njem & $253-259$ & Ostrakon \\
\cline { 2 - 5 } & O.BuNjem 27 & Bu Njem & $253-259$ & Ostrakon \\
\cline { 2 - 5 } & O.BuNjem 60 & Bu Njem & $253-259$ & Ostrakon \\
\cline { 2 - 5 } & ChLA X 443 & Unbekannt & 3. Jahrhundert & Papyrus \\
\hline
\end{tabular}

\section{Conclusio}

Zusammenfassend lässt sich festhalten, dass die Spuren und Hinweise zur medizinischen Versorgung im römischen Heer zumeist indirekt sind, wobei das Schweigen der Quellen nicht auf ein Fehlen der Wundversorgung und Verwundetenfürsorge hindeutet, sondern im Gegenteil dafür spricht, dass es sich dabei um Routinetätigkeiten gehandelt hat, die nicht gesondert in militärischen Berichten oder Briefen an die Familien erwähnt zu werden brauchten. Der Stand der ärztlichen Betreuung in römischen Militär scheint gut gewesen zu sein; es gibt keine Hinweise darauf, dass Soldaten für die medizinische Versorgung zahlen mussten; es sind auch keine Klagen von Soldaten über schlechte medizinische Behandlung überliefert - insofern bemerkenswert, als sich bereits die römischen Soldaten über alles und jeden beschwerten; dass auch aus dem in der Kaiserzeit ausgesprochen friedlichen und so dokumentenreichen Ägypten keinerlei Beschwerden bekannt sind, ist noch einmal viel bemerkenswerter. ${ }^{27}$ Mit nur einer Ausnahme sind alle lateinischen Dokumente, die sich mit kranken Soldaten und der militärischen Versorgung von Kranken und Verwundeten direkt oder indirekt befassen, Listen; lediglich C.Epist.Lat. I 233 als Teil des Personalaktes des Sarapion bildet mit seiner brieflichen inscriptio eine Ausnahme. Sowohl im westlichen wie im östlichen Teil des Römischen Reiches ist Latein die offizielle militärische Sprache - das spiegelt sich auch in den hier behandelten offiziellen

${ }^{27}$ Wesch-Klein, Aspekte, 82. 
militärischen Dokumenten wieder. Der primäre Zweck der Texte, die Licht auf die römische militärmedizinische Versorgung und die Berichterstattung über den medizinischen Zustand der Soldaten werfen, ist ein militärischer, kein medizinischer. Umso mehr fällt ins Auge, dass zumindest in den Kategorien B1 und B2 genauere pathologische Angaben gemacht werden. Ähnlichkeiten in Aufbau und Inhalt lassen sich mit griechischen Dokumenten vom Mons Claudianus festhalten; allerdings stammen diese nicht aus dem militärischen Umfeld, wohl aber aus dem der kaiserlichen familia. ${ }^{28}$ Aus dem direkten militärärztlichen Umfeld, vermutlich der offiziellen Buchhaltung, ist bislang lediglich ein Dokument bekannt, P.Masada 723 (Kategorie A).

\section{Literaturverzeichnis}

Bernini, Fasce $=$ Andrea Bernini, Fasce di lana in un papiro latino (P.Masada 723 riedito), APF 64 (2018), 312-23.

Cotton/Geiger, Masada II = Hannah M. Cotton / Joseph Geiger, Masada II, The Yigael Yadin Excavations 1963-1965, Final Reports. The Latin and Greek Documents, Jerusalem 1989.

Davies, Medici = Roy W. Davies, Some More Military Medici, „Epigraphische Studien“ 9 (1972), 1-11.

Domaszewski/Dobson, Rangordnung $=$ Alfred von Domaszewski / Brian Dobson, Die Rangordnung des römischen Heeres. 2. durchgesehene Auflage. Einführung, Berichtigung und Nachträge von Brian Dobson, Köln/Graz 1967.

Draycott, Approaches = Jane L. Draycott, Approaches to Healing in Roman Egypt, Oxford 2012.

Graß1, Missio = Herbert Graßl, Missio causaria, in: E. Weber / G. Dobesch (Hrsg.), Römische Geschichte, Altertumskunde und Epigraphik. Festschrift für Artur Betz zur Vollendung seines 80. Lebensjahres, Wien 1985, 281-9.

Hirt Raj, Médecins = Marguerite Hirt Raj, Médecins et maladies de l'Egypte romaine. Étude socio-légale de la profession médicale et de ses praticiens du Ier au IVe siècle ap. J.-C., Leiden/Boston 2006.

Israelowich, Healers = Ido Israelowich, Patients and Healers in the High Roman Empire, Baltimore 2015.

Kaiser, Evidenz = Anna M. Kaiser, Die papyrologische Evidenz zur medizinischen Versorgung im römischen Militär, in: R. Breitwieser / F. Humer / E. Pollhammer / R. Arnott (Hrsg.), Medizin und Militär - Soldiers and Surgeons. Beiträge zur Wundversorgung und Verwundetenfürsorge im Altertum, Bad Vöslau 2018, 120-9.

Kaiser, Routinedienste $=$ Anna M. Kaiser, Alltägliche Routinedienste römischer Soldaten, in: P. Mauritsch (Hrsg.), Akten des 13. Österreichischen Althistorikerinnen- und Althistorikertages, 18.-20. November 2010, Graz 2011, 115-29.

Krause, Versorgung = Olaf Krause, Die medizinische Versorgung im römischen Legionslager Vindonissa. Valetudinarium, Inschriften und Instrumente, in: P. Jung / N. Schücker (Hrsg.), Utere felix vivas. Festschrift für Jürgen Oldenstein, Bonn 2012, 159-70.

Netzer, Masada III = Ehud Netzer, Masada III, The Yigael Yadin Excavations 1963-1965, Final Reports. The Buildings Stratigraphy and Architecture, Jerusalem 1991.

${ }^{28}$ Vgl. z.B. Serafino, Familia. 
Palme, Legionäre = Bernhard Palme (Hrsg.), Die Legionäre des Kaisers. Soldatenleben im römischen Ägypten, Wien 2011.

Premerstein, Buchführung = Anton von Premerstein, Die Buchführung einer ägyptischen Legionsabteilung, „Klio“ 3 (1903), 1-46.

Salazar, Treatment $=$ Christine F. Salazar, The Treatment of War Wounds in Graeco-Roman Antiquity, Leiden/Boston/Köln 2000.

Scheidel, Rekruten $=$ Walter Scheidel, Rekruten und Überlebende: Die demographische Struktur der römischen Legionen in der Prinzipatszeit, „Klio“ 77 (1995), 232-54.

Serafino, Familia = Cristina Serafino, Familia e paganoi al Mons Claudianus, in: E. Lo Cascio / G. D. Merola (Hrsg.), Forme di aggregazione nel mondo romano, Bari 2008, 293-301.

Speidel, Schreibtafeln $=$ Michael A. Speidel, Die römischen Schreibtafeln von Vindonissa . Lateinische Texte des militärischen Alltags und ihre geschichtliche Bedeutung, Brugg 1996.

Speidel, Einheit = Michael A. Speidel, Einheit und Vielfalt in der römischen Heeresverwaltung, in: M. A. Speidel (Hrsg.), Heer und Herrschaft im Römischen Reich der Hohen Kaiserzeit, Stuttgart 2009, 283-304.

Stauner, Schriftwesen $=$ Konrad Stauner, Das offizielle Schriftwesen des römischen Heeres von Augustus bis Gallienus (27 v. Chr.-286 n. Chr.): Eine Untersuchung zu Struktur, Funktion und Bedeutung der offiziellen militärischen Verwaltungsdokumentation und zu deren Schreibern, Bonn 2004.

Wesch-Klein, Aspekte $=$ Gabriele Wesch-Klein, Soziale Aspekte des römischen Heerwesens der Kaiserzeit, Stuttgart 1998.

Wilmanns, Rangordnung $=$ Juliane C. Wilmanns, Zur Rangordnung der römischen Militärärzte während der mittleren Kaiserzeit, ZPE 69 (1987), 177-89.

Wilmanns, Sanitätsdienst $=$ Juliane C. Wilmanns, Der Sanitätsdienst im Römischen Reich, Hildesheim/Zürich/New York 1995.

Yadin, Excavation = Yigael Yadin, The Excavation of Masada - 1963/64: Preliminary Report, „Israel Exploration Journal“ 15 (1965), 1-120. 